\title{
Veinte años de protección de cauces en el litoral de la Región de Murcia (1994-2014). Evaluación de la eficiencia de las medidas de Ordenación del Territorio
}

\author{
Alfredo PÉREZ MORALES \\ Departamento de Geografía \\ Universidad de Murcia \\ alfredop@um.es \\ Salvador GIL GUIRADO \\ salvador.gil@um.es \\ Fernando M. GARCÍA MARTÍN \\ Departamento de Arquitectura y Tecnología de la Edificación \\ Universidad Politécnica de Cartagena \\ fernando.garcia@upct.es
}

Recibido: 17 de diciembre de 2014

Enviado a evaluar: 18 de diciembre de 2014

Aceptado: 27 de abril de 2015

\section{RESUMEN}

En las últimas décadas se ha producido un incremento significativo de los episodios de inundación en el litoral de la Región de Murcia que hace cuestionar el papel desempeñado por las estrategias preventivas de ordenación. En este trabajo se evalúa la eficiencia de las medidas de protección de cauces incluidas en las Directrices y Plan de Ordenación del Litoral de la Región de Murcia. Para ello se utiliza la información catastral y la modelización hidrológica del periodo de retorno de 10, 50, 100 y 500 años. Los resultados demuestran que la exposición al peligro ha seguido aumentando incluso después de la aprobación de dicho instrumento y nos permiten evidenciar al aumento de la exposición y la vulnerabilidad como factores principales en dicha acentuación de las pérdidas económicas asociadas a inundaciones.

Palabras clave: Inundación, Exposición, Catastro, Periodo de Retorno, Ordenación del Territorio. 


\title{
Twenty years of protection of rivers on the coast of the Region of Murcia (1994-2014 ). Evaluation of the efficiency of the measures of Planning
}

\begin{abstract}
A significant increase in episodes of flooding on the coast of Murcia occurred in last decades, questioning the role of preventive strategies on planning. In this paper is evaluated the efficiency of river channel protection actions included in the Guidelines and Planning of Región de Murcia Coast. The cadastral information and the hydrological modeling of 10, 50, 100 and 500 years of return period have been used to study the evolution of vulnerability. The results shows that exposure to risk has continued to increase even after the adoption of these guidelines, becoming a key factor in the increase of economic losses associated with flooding.
\end{abstract}

Key words: Flood, Exposure, Land Registry, Return Period, Land Management.

\author{
Vingt ans de protection des rivières sur \\ la côte de la région de Murcie (1994-2014). \\ Évaluation de l'efficacité des mesures de planification
}

\section{RÉSUMÉ}

$\mathrm{Au}$ cours des dernières décennies, on a pu constater un accroissement significatif des épisodes d'innodation au long du littoral de la région de Murcia. Cet accroissement met en question le rôle des stratégies préventives d'ordonnance. Ce travail évalue l'efficacité des mesures de protection des lits inclues dans les directrices et le plan d'ordonnance du littoral de la région de Murcia. À cet effet, on utilise l'information cadastrale et la modélisation hydrologique de la période de retour de 10, 50, 100 et 500 ans. Les résultats prouvent que l'exposition au danger a continué à augmenter même après l'approbation de tel instrument et nous permettent de mettre en évidence l' exposition croissante et la vulnérabilité comme principaux facteurs de cette accentuation des pertes économiques associées aux innondations.

Mots clés: Inondation, Exposition, Cadastre, Période de retour, Amenagement du Territoire.

\section{INTRODUCCIÓN}

El espacio geográfico integrado por el litoral de la Región de Murcia constituye una de las unidades naturales más peculiares de la misma por sus rasgos geológicos, climáticos y paisajísticos. Estos aspectos hacen de este territorio un foco de atención social, político y económico que motivaron la aparición de varias iniciativas de carácter planificador con más o menos suerte en su aplicación (Pérez, 2007). De todas ellas sobresalen las vigentes Directrices y Plan de Ordenación Territorial del Litoral de la Región de Murcia (DPOTL de ahora en adelante), de 18 de junio de 2004. Se trata de una iniciativa que nace al amparo de la Ley $1 / 2001$, de 24 de abril, del Suelo de la Región de Murcia y la Ley 6/1998, de 13 de abril, sobre régimen del suelo y 
valoraciones, en un contexto socioeconómico en el que la bajada progresiva del precio del dinero y la consolidación del turismo residencial vinculado a los propietarios foráneos (españoles y extranjeros), animaron el ya de sobra conocido boom inmobiliario que se mantuvo hasta finales de 2007 (Gaja, 2008; Burriel, 2008).

En este sentido, el gobierno regional, lejos de elaborar un instrumento capaz de organizar ese crecimiento desaforado de una forma acorde con las limitaciones ambientales del ámbito en cuestión, trató de sumarse a la dinámica del desarrollismo de la manera más flagrante mediante la aprobación de las mencionadas directrices. En concreto, las DPOTL abrían claras oportunidades liberalizadoras al fomento de los complejos residenciales y actuaciones integrales en turismo como la macrourbanización de Marina de Cope (Vera y Espejo, 2006). En consecuencia, la intensificación de la ocupación de espacios que hasta entonces no habían tenido una vocación más allá de la agrícola, provocará un aumento de la exposición de dichas edificaciones al peligro de inundación sin precedentes en que, con el paso de los años, las avenidas han ido evidenciando (Gil-Guirado et al., 2014).

Figura 1. Mapa del área de estudio y representación de la zona inundable y parcelas catastrales.

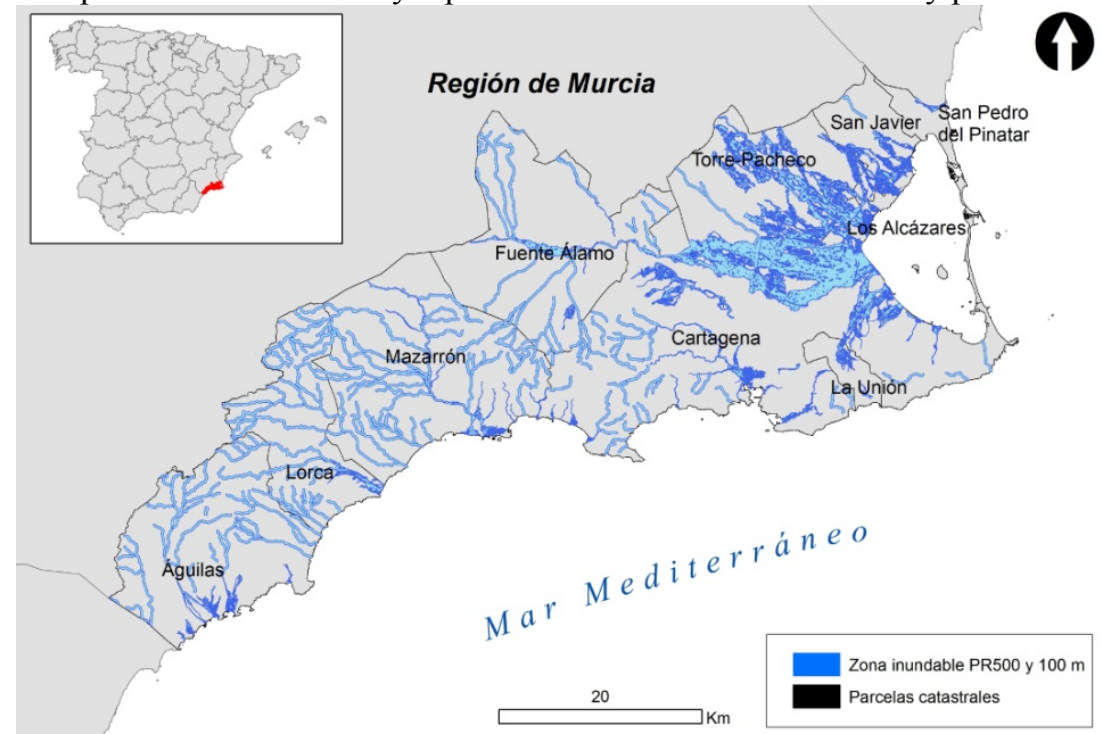

Fuente: Elaboración propia.

A este respecto, hay que señalar que las DPOLT se aprueban en un momento en el que existía cierta confusión en cuanto a la competencia para proteger cauces de su ocupación (Olcina, 2007). Por tanto, lo que habría sido una oportunidad inmejorable de sentar las bases de organización coherente con lo relativo al respeto del trazado de las ramblas y barrancos que drena el litoral de la Región de Murcia, se resuelve con dos breves artículos que inclinan a dudar sobre su eficiencia: 
Art. 10.-El suelo de protección de cauces está formado por una banda de $100 \mathrm{~m}$. a ambos lados de los cauces, medida en los márgenes que se reflejan en la cartografía regional 1:5000.

- No estarán incluidos en esta categoría los suelos urbanos y urbanizables sectorizados que tengan dicha clasificación a la entrada en vigor de dicho documento.

Art. 11.- La realización de un estudio de inundabilidad y su aprobación por la administración competente, permitirá la modificación de los $100 \mathrm{~m}$. de protección a aquellos nuevos límites que marque dicho estudio. Estos estudios se realizarán de acuerdo con la normativa que a tal efecto desarrolle, la Comunidad Autónomas, pudiéndose llevarse a cabo a través del Ayuntamiento por cuyo término municipal discurra el tramo del cauce o a instancia de parte, o bien por la Consejería competente.

Ante esta situación, y una vez han transcurrido diez años desde la aprobación de las DPOTL, interesa saber su validez en cuanto a la protección que hayan podido ejercer con respecto a los procesos de ocupación materializado en el decurso de ese periodo. Por ende, el principal objetivo del presente trabajo es el de evaluar el grado de exposición dentro del ámbito reglado por las DPOTL. Afortunadamente este tipo de estudios centrados en el análisis del factor humano anteriormente se realizados con la evaluación de superficies por usos del suelo (Moel et al., 2009), han sido mejorados sustancialmente (Jongman et al, 2012) gracias a los avances en modelización hidrológica y, sobre todo, la digitalización del espacio construido por parte de los organismos oficiales. Mediante estas dos fuentes de información espacial, hoy se puede llevar a cabo con cierta solvencia soluciones que nos permiten evidenciar el grado, la intensidad y el volumen de lo expuesto en áreas propensas a experimentar inundaciones con una precisión mejorada.

\section{2. ÁREA DE ESTUDIO}

El ámbito de actuación de las DPOTL abarca el término administrativo de todos los municipios costeros de la Región de Murcia, es decir: Águilas, Mazarrón, Cartagena, La Unión, Los Alcázares, San Javier y San Pedro del Pinatar. A estos se añaden los municipios prelitorales de Fuente Álamo y Torre Pacheco por la intensidad de sus relaciones con los primeros, y su creciente dinamismo socioeconómico. En total conforman un área que alcanza los $1.893,90 \mathrm{~km}^{2}$ y que representa un total de $16,74 \%$ con respecto a la superficie de la Región de Murcia. En líneas generales se trata de un área donde los factores y elementos climáticos predominantes, la disposición orográfica, las pendientes y la naturaleza impermeable del suelo configuran un tipo de red hidrográfica que padece largos periodos de estiaje $y$ que entra solo en funcionamiento en situaciones de lluvia de fuerte intensidad 
horaria generando crecidas e inundaciones del tipo flash-flood. En otras palabras, lo anterior configura una situación de vulnerabilidad biofísica (Cutter et al., 2003) que ha sido malinterpretada y ocasiones ni siquiera percibida por los agentes responsables de los procesos de ocupación contribuyendo a la creación de un escenario de riesgo.

En este contexto más o menos generalizado por todo el litoral Mediterráneo español, cada vez que acontece una avenida de rango extraordinario, lo hace provocando inundaciones con efectos negativos sobre la población, actividades y edificaciones. De hecho, tal y como señalan Gil-Guirado et al. 2014, ese umbral de precipitaciones a partir del cual se producen problemas es cada vez menor. La figura 2 elaborada por los mismos autores, confirma lo anterior, pues en ella se advierte cómo el número de episodios de inundación de mayor o menor intensidad han ido acentuándose con el paso del tiempo en el área de estudio. Si consideramos el escenario de desarrollo urbano hasta ahora señalado, parece evidente que ese incremento en el número de inundaciones venga explicado por una mala adaptación practicada por los procesos de ordenación y ocupación más que por unos cambios en la dinámica atmosférica.

Figura 2. Número anual de inundaciones en el área de estudio (1900-2013).

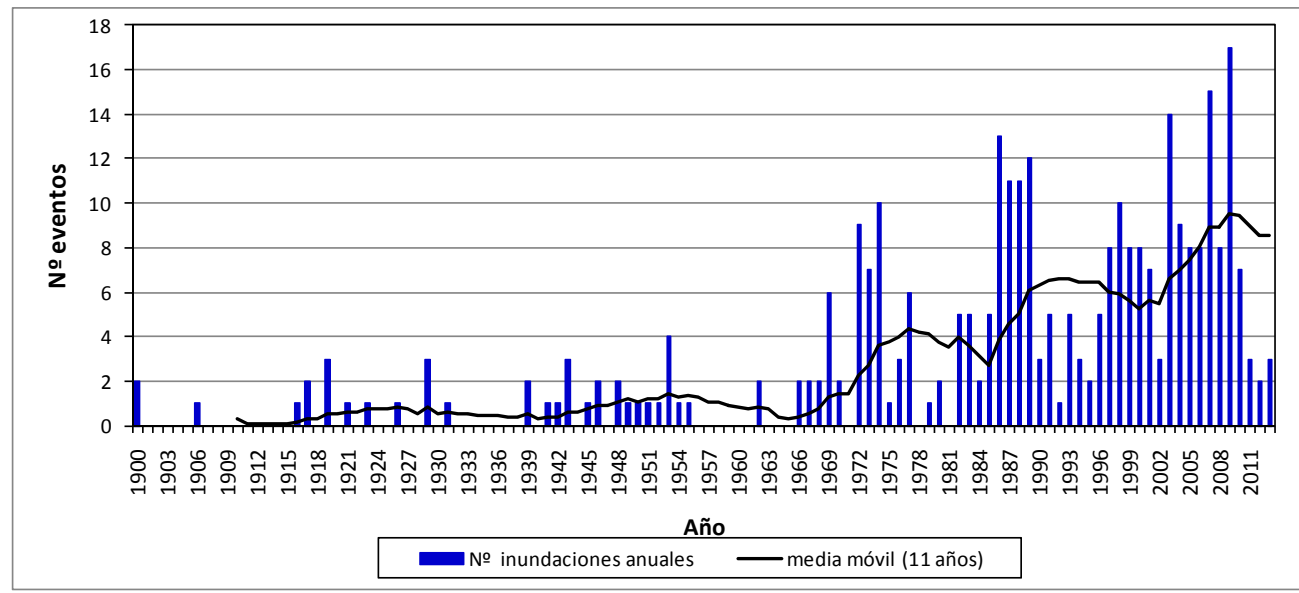

Fuente: Gil-Guirado et. al. 2014.

Ante esta situación, conviene evaluar dichas estrategias de carácter preventivo como son las que se incluyen en la DPOTL a fin de diagnosticar el nivel de vulnerabilidad que presentan, y buscar mejoras que puedan implementarse para corregir los desajustes que los procesos naturales como son las inundaciones ponen en evidencia cada vez que tienen lugar. 


\section{METODOLOGÍA Y FUENTES}

Para la evaluación de la exposición frente a inundaciones, es necesaria la consideración espacial de dos de los factores esenciales, el componente físico o peligro y el humano (Cardona et al., 2012). El primero, se obtuvo de la información contenida en el Sistema Nacional de Cartografía de Zonas Inundables (SNCZI) (MAGRAMA, 2014). Se trata del instrumento oficial elaborado por el Ministerio de Agricultura, Alimentación y Medio Ambiente que, siguiendo los principios de la Directiva 2007/60 sobre evaluación y gestión de riesgos de inundación, se emplea en la gestión del espacio fluvial, la prevención de riesgos y la planificación territorial. La citada fuente proporciona los resultados de las modelizaciones hidrológicas en formato vectorial para los siguientes periodos de retorno: Periodo de Retorno de 10 años (PR10); 50 años (PR50); 100 años (PR100); y 500 años (PR500). Una vez situados sobre el terreno se confecciona el mapa de zonas inundables que se empleó para el presente estudio.

Para el componente humano, se consideraron las edificaciones en forma de parcelas catastrales ${ }^{1}$ siguiendo la metodología propuesta por García (2013). Ésta información se obtuvo de la sede electrónica de la Dirección General del Catastro, dependiente del Ministerio de Hacienda y Administraciones Públicas con datos actualizados a Mayo de 2013 (MHAP, 2014). Los productos catastrales que se consultaron fueron los datos de naturaleza urbana ${ }^{2}$ y las parcelas para cada municipio del área de estudio. Los datos catastrales vienen en formato alfanúmerico, e incluyen toda la información del catastro excepto la sujeta a confidencialidad. La información se organiza en una sucesión de registros con tamaño individual de 1000 caracteres. Cada uno de esos registros contiene información codificada entre la que se encuentra el año de construcción de cada unidad o parcela catastral. Por su parte, la cartografía está compuesta por una serie de polígonos que representan las mencionadas parcelas catastrales. Los polígonos vienen acompañados de una tabla de atributos con información detallada sobre las características de los mismos e identificados mediante

${ }^{1}$ La parcela es la unidad de desagregación máxima utilizada en este trabajo y que corresponde, por regla general, a cada edificación localizada sobre el terreno.

2 Siguiendo la distinción de tipos de bienes inmobiliarios según la naturaleza del suelo establecida en el RDL 1/2004 por el que se aprueba el texto refundido de la Ley del Catastro Inmobiliario (BOE 8/03/2004), las descargas de cartografía pueden realizarse de los suelos de naturaleza urbana de los de naturaleza rústica. De acuerdo a los artículos 6,7 y 8 de dicho texto refundido, se entiende como suelo de naturaleza urbana, de manera simplificada, "el clasificado o definido por el planeamiento urbanístico como urbano, urbanizado o equivalente" y "los que tengan consideración de urbanizable". El suelo de naturaleza rústica es "aquel que no sea de naturaleza urbana, ni esté integrado en un bien inmueble de características especiales". Esta categoría especial incluye los suelos que "constituyen un conjunto complejo de uso especializado" perteneciente a alguno de los siguientes grupos: destinados a producción energética (refinerías, embalses, etc.), vías de circulación (autopistas, carreteras y túneles de peaje) o aeropuertos y puertos comerciales. 
el campo referencia catastral. Gracias a ese registro oficial de cada parcela (referencia catastral), todos los datos referentes a la antigüedad de lo construido anteriormente señalados, se incorporan a la tabla de atributos correspondiente a los polígonos que representan las parcelas catastrales mediante el gestor de la base de datos de un SIG y se obtiene el mapa del factor humano.

El trabajo señalado hasta ahora nos permite culminar la confección del mapa de exposición gracias a la combinación de las dos componentes principales, peligro y parcelas. Este valioso documento nos ayuda a determinar la localización de lo expuesto a una inundación potencial según su probabilidad de ocurrencia por periodos de retorno y a evaluar, con precisión métrica, las superficies construidas potencialmente afectables. Gracias a ese nivel de detalle se ha podido reducir el denominador de la escala de trabajo hasta un grado de exactitud que permite manejar información a nivel cartográfico de plano, es decir, por debajo de la escala 1:5000. Se trata de mapas perfectamente aplicables para cualquier tarea administrativa y que facilitan la obtención de otro tipo de salidas cartográficas más complejas como el mapa de riesgos naturales que no son objeto del presente estudio.

A pesar de la bondad de las fuentes manejadas, se ha tenido que hacer frente en nuestro análisis a una serie de limitaciones asociadas a la propia naturaleza y características de las mismas. En lo referente a los datos catastrales asociados a parcelas, se ha detectado que, en algunos casos, una misma parcela catastral puede contener más de una construcción edificada en distintos años. En esos casos se tomó el año de la construcción más antiguo evitando una infraestimación del número de parcelas edificadas en los primeros años de la serie y asumiendo que las construcciones dentro de esas parcelas realizadas en otros años se tratan de obras de remodelación o ampliación de la edificación existente. Se ha calculado el número de parcelas con construcciones realizadas en distintos años para evaluar el error máximo que podría significar este criterio (si fuese equivocado en todos los casos) tanto en las edificaciones expuestas como en las que no lo están y que se resumen en la tabla 1.

Tabla 1. Porcentaje de parcelas que contienen más de una construcción y el total de parcelas catastrales del área de estudio diferenciadas entre las expuestas y las no expuestas.

\begin{tabular}{|c|c|c|c|}
\cline { 2 - 3 } \multicolumn{1}{c|}{} & \multicolumn{2}{c|}{ Parcelas con más de 1 construcción } & \multirow{2}{*}{ Total Parcelas } \\
\cline { 2 - 3 } \multicolumn{1}{c|}{} & Total & $\%$ & \\
\hline No expuesto & 4.200 & $5,94 \%$ & 70.695 \\
\hline Expuesto & 1.259 & $5,14 \%$ & 24.494 \\
\hline
\end{tabular}

Fuente: Elaboración propia. 
En segundo lugar, al igual que sucede en otros trabajos de similares características (Jongman et al., 2014), el hecho de no disponer de modelizaciones hidrológicas anteriores a las empleadas en nuestro análisis, se asume que las zonas inundables de los periodos de retorno han permanecido estables a lo largo del tiempo. Sin embargo, en el ámbito geográfico analizado las transformaciones antrópicas de los cauces fluviales han sido intensas y a buen seguro habrán alterado las zonas inundables en ese periodo (Bochet y Garía-Fayos, 2004; Hooke, 2006; Symeonakis et al., 2007)

Durante el desarrollo metodológico para la obtención del mapa de exposición se ha detectado una seria dificultad a la hora de evaluar con precisión la superficie ocupada en zona inundable. En ocasiones, debido a la mejora en la precisión de las modelizaciones hidrológicas, la zona inundable sigue el trazado de las calles del núcleo urbano y la lámina de agua modelizada no se solapa con el de las parcelas asociadas a edificaciones, por lo que no existe una intersección posible con la zona inundable para detectar las propiedades que resultarían afectadas pese a que hay constancia de que las partes bajas de esos edificios son las primeras que sufren los impactos ante una inundación potencial (Gil-Guirado et al, 2014). Por tanto, se ha considerado que esos inmuebles pasarían a formar parte de la superficie inundable, realizando para ello un buffer de 1 metro sobre periodo de retorno.

Por último habría que destacar las cuestiones relativas a la fiabilidad de las modelizaciones hidrológicas e hidráulicas en las que se apoya la delimitación de los inmuebles expuestos. El cálculo de los periodos de retorno presenta un problema clásico y es el de los valores extremos que se presentan en el análisis de series de datos. Aunque muchas modelizaciones en las que se ha basado este trabajo consideran estos valores extremos, la contrastación con datos empíricos, raramente, por falta de registros suficientemente largos supera los 100 años de forma fiable en la mayor parte de los casos, por lo que está práctica puede resultar científicamente criticable al basarse en la mera suposición de que la Naturaleza deba necesariamente ajustarse a una formulación matemática teórica, especialmente cuando el periodo de retorno asignado con este sistema supera el doble de la longitud temporal de la serie analizada (Olcina, 2007). Algunos avances en este sentido se están alcanzando, Balasch et al. (2014) han calculado para la Cuenca del Río Ebro una infraestimación de los periodos de retorno con respecto a la reconstrucción de caudales de inundaciones históricas de más del $25 \%$, si bien estás datos también son de reconstrucciones y tampoco ofrecen un valor real. En consecuencia a pesar del buen trabajo realizado en el SNCZI, se hace necesaria una mayor consideración de series empíricas más largas que permitan el ajuste correcto de esos máximos (Barriendos et al, 2014). De aplicarse en el área de estudio es probable que la zona expuesta incluso aumentara sus dimensiones.

\section{RESULTADOS}

El incremento en el volumen de pérdidas económicas vinculadas a los episodios de inundación en las últimas décadas ha estado motivado por el aumento del nivel de vida, propio de las sociedades occidentales. Los cambios socioeconómicos experimentados en España han tenido una especial repercusión sobre el territorio y, 
según los datos, sobre la exposición a los peligros naturales (Barredo et al., 2012). Proceso especialmente intenso y acusado en el ámbito litoral, donde el crecimiento urbano reciente ha sido calificado como un auténtico "tsunami urbanizador" (Gaja, 2008) que, lejos de seguir una ocupación ordenada del espacio, ha materializado un escenario de riesgo que no ha sido controlado.

Figura 3. Curvas de saturación de la superficie construida (izquierda) y el número de inmuebles (derecha) acumulados en las zonas inundables de los distintos periodos de retorno.

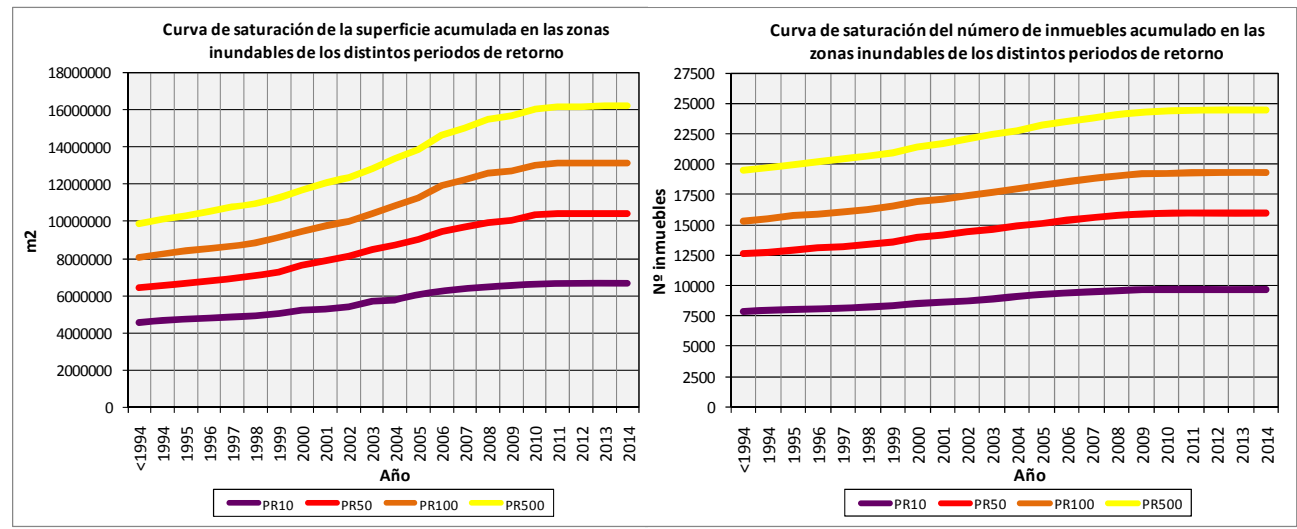

Fuente: Elaboración propia.

De acuerdo a los resultados de la figura 3, desde 1994, año de la aprobación de las DPOTL, la superficie urbanizada y el número de inmuebles construidos en la zona inundable delimitada por los diferentes periodos de retorno ha ido experimentando un crecimiento acumulado continuo en el litoral de la Región de Murcia que supera las 4.979 edificaciones y un total de 6.315.640,62 $\mathrm{m}^{2}$ (PR500). En cifras relativas los resultados son verdaderamente alarmantes. Para el periodo analizado el incremento de las parcelas urbanas es del $25,5 \%(<1994=100)$, lo que supone una tasa de crecimiento anual del $1,3 \%$. Sin embargo, llama la atención que el aumento de las parcelas urbanas en áreas propensas a experimentar inundaciones ha sido mayor que el registrado en áreas no inundables $(24,7 \%)$. En lo que se refiere a la superficie ocupada por edificaciones en terrenos expuestos, los valores son sensiblemente inferiores $(63,8 \%)$ con respecto a lo que ha sucedido en zonas no expuestas $(79,7 \%)$. En cualquier caso no dejan de ser cifras muy elevadas que dan una idea del proceso acelerado de expansión urbanística y de la laxitud en la aplicación o generación de normativas de mitigación del riesgo de inundación como son las DPOTL. 
Tabla 2. Resumen de las edificaciones construidas en zonas expuestas y no expuestas al peligro de inundación en el área de estudio para el periodo anterior y posterior a la aprobación de las DPOTL.

\begin{tabular}{|c|cc|cc|cc|cc|}
\cline { 2 - 9 } \multicolumn{1}{c|}{} & \multicolumn{4}{c|}{ Edificaciones expuestas } & \multicolumn{3}{c|}{ Edificaciones no expuestas } \\
\cline { 2 - 10 } \multicolumn{1}{c|}{} & \multicolumn{2}{|c|}{ Superficie } & \multicolumn{2}{c|}{ Edificaciones } & \multicolumn{2}{c|}{ Superficie } & \multicolumn{2}{c|}{ Edificaciones } \\
\hline Años & $(\mathrm{m} 2)$ & $(\%)$ & $\left(\mathrm{n}^{\circ}\right)$ & $(\%)$ & $(\mathrm{m} 2)$ & $(\%)$ & $\left(\mathrm{n}^{\circ}\right)$ & $(\%)$ \\
\hline$<\mathbf{1 9 9 4}$ & $9.889 .529,4$ & $31,9 \%$ & 19.515 & $25,7 \%$ & $21.051 .088,3$ & $68,1 \%$ & 56.448 & $74,3 \%$ \\
\hline $\mathbf{1 9 9 4 - 2 0 0 3}$ & $2.950 .872,2$ & $26,4 \%$ & 2.906 & $27,4 \%$ & $8.224 .043,7$ & $73,6 \%$ & 13.947 & $73,6 \%$ \\
\hline $\mathbf{2 0 0 4 - 2 0 1 4}$ & $3.364 .768,4$ & $28,1 \%$ & 2.073 & $24,9 \%$ & $8.568 .526,7$ & $71,8 \%$ & 6.251 & $75,1 \%$ \\
\hline TOTAL & $16.205 .170,04$ & & 24.494 & & $37.843 .658,4$ & & 70.395 & \\
\hline
\end{tabular}

Fuente: Elaboración propia.

Si analizamos los datos en dos periodos iguales antes y después de la aplicación de las DPOTL (1994-2003 y 2004-2014) parece que ese incremento de lo construido en zona inundable con respecto a lo no expuesto se ha debilitado ligeramente en el segundo periodo (de $27,4 \%$ ha pasado al $24,9 \%$ ) y, por lógica, lo edificado en zona no expuesta ha enjugado ese descenso ganando mayor protagonismo (del $72,5 \%$ al $75,1 \%$ (ver tabla 2). Se podría aventurar que una de las razones de esta tendencia es que las medidas de conservación y limitación de usos aplicadas tras la aprobación de las DPOTL en 2004 comenzaron a ejercer su papel. No obstante, de ser así, la eficiencia de las mismas deja bastante que desear al no haber contenido la ocupación de lechos de inundación, incluso en circunstancias donde la crisis financiera ha supuesto una ralentización de la presión inmobiliaria en zonas supuestamente protegidas ante la mayor disponibilidad de suelo útil.

La figura 4 nos permite distinguir una serie de cuestiones que nos ayudan a entender las cifras antes señaladas de forma más detallada. La superación de la crisis económica que afectó a España en 1993 y que redujo la actividad inmobiliaria de manera drástica, supuso el inicio progresivo del segundo periodo de incremento más acentuado vivido en España tras el acontecido desde los años sesenta hasta 1993. Entre 1994 y 1997 se inicia un nuevo despunte de la construcción, pero es sobre todo a partir de 1998 cuando se desató, una vez más, un proceso de edificación desaforada que, lejos de respetar el límite de los cauces volvió a contribuir al aumento y acumulación tanto de edificios, como de superficie ocupada en zonas inundables. Entre 1998 y 2006, se levantaron 2.150 nuevas edificaciones (63,5\% de lo construido entre 1994 y 2014) con una tasa de edificación en ámbito expuesto casi tan acentuada como en el no expuesto. Posteriormente, se produce el descenso acusado de los valores que atañen a lo construido y que revelaron la crisis del sector que experimentamos en la actualidad. 
Figura 4. Evolución de la superficie construida en zona expuesta en los diferentes periodos de retorno y de la superficie no expuesta (en discontinua) durante el periodo 1994-2014.

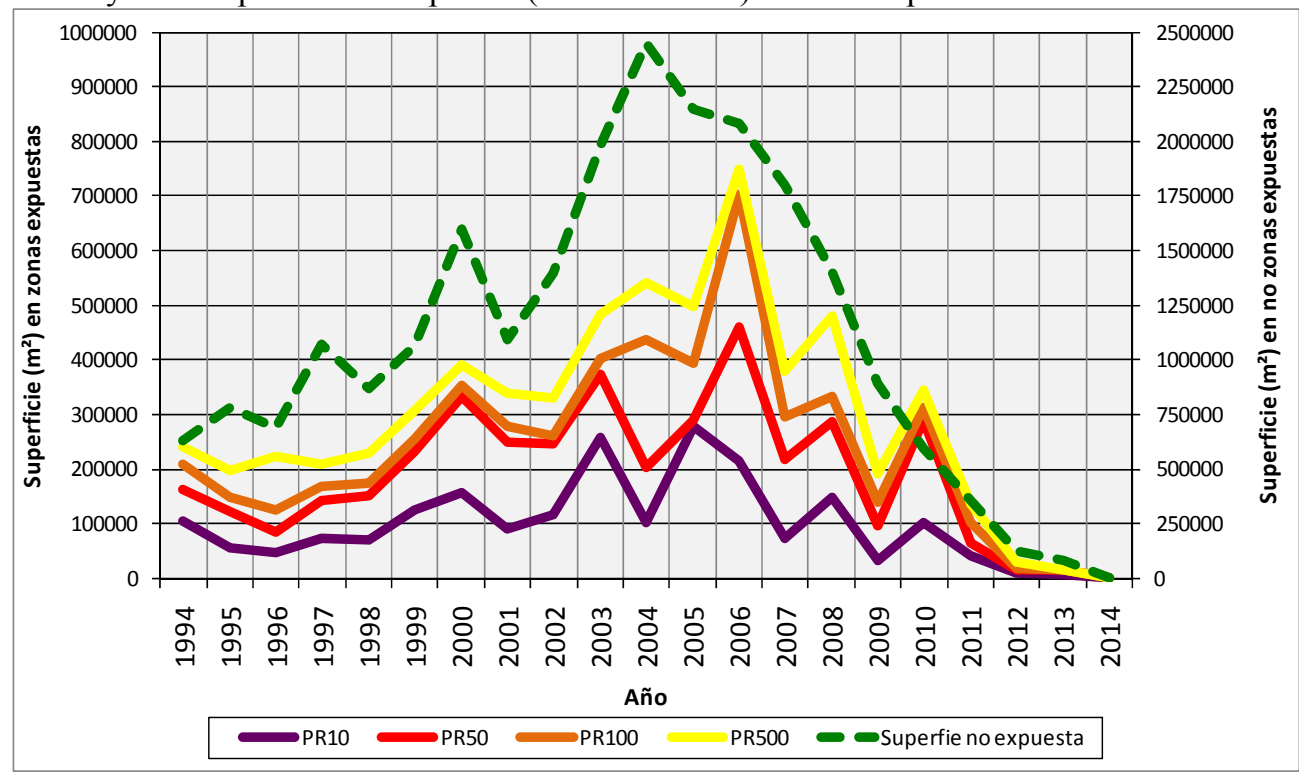

Fuente: Elaboración propia.

Entre las causas que ayudan a entender la magnitud del fenómeno analizado hasta ahora destaca la propia liberalización del proceso urbanizador experimentado con la aprobación estatal de la Ley del Suelo de 1998 y de algunas leyes autonómicas y de planificación urbana municipales en el ámbito mediterráneo como las DPOTL (Jiménez, 2009). En algunos municipios (ej. Los Alcázares) la promoción de suelo para su urbanización ha agotado, prácticamente, este recurso. De otro lado, la demanda de viviendas para residencias de inmigrantes europeos fue creciente en el área de estudio, en un contexto de bonanza económica en Europa en esos años. Se trata de un grupo poblacional que, atraído por las condiciones climáticas invernales de las tierras mediterráneas $\mathrm{y}$, sobre todo, una coyuntura económica ventajosa en cuanto a los bajos tipos de interés en los préstamos hipotecarios en España (se pasó del $16,6 \%$ en diciembre de 1990 al 4,2\% a mediados de 2006, con valores mínimos del 3,19 en agosto de 2005) llegaron de forma masiva al mediterráneo español para adquirir segundas residencias. 
Figura 5. Evolución del aumento de la exposición en dos núcleos urbanos del área de estudio: Torre-Pacheco y San Javier.
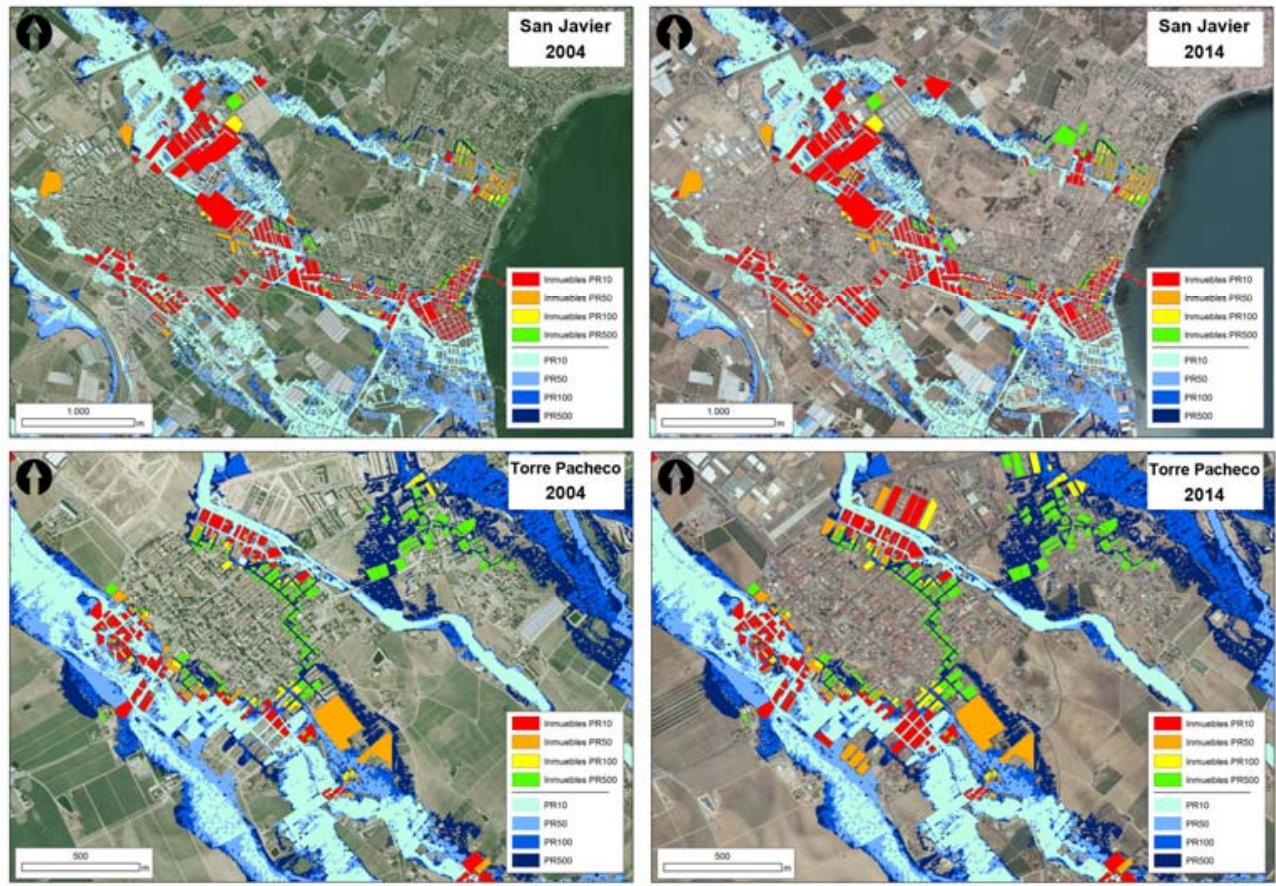

Fuente: Elaboración propia.

Espacialmente, el resultado de este proceso de expansión del casalicio en ámbitos inundables durante los 20 años analizados se ha traducido en la sucesiva integración en el callejero urbano de cursos fluviales, y por ende, el incremento progresivo de la exposición y el riesgo de inundación (ver figura 4). Lo anterior puede advertirse en la distribución de lo construido en los distintos periodos de retorno con respecto al total (ver figura 6). La figura 6 señala que hasta estos últimos años la ocupación de lo inundable se ha hecho principalmente sobre la zona de los 10 años. Se trata de un dato alarmante, que resulta de un comportamiento corriente en un ámbito geográfico donde la red hidrográfica está compuesta en su mayoría por ramblas y barrancos que de forma natural permanecen secos durante largos periodos de tiempo, y tan solo ven interrumpida esta condición en situaciones transitorias tras lluvias de fuerte intensidad horaria, contribuyendo esto a una baja percepción del peligro de inundación (Llasat et al, 2008). Desafortunadamente, estas características físicas han invitado desde siempre a ocupar las márgenes más próximas a los lechos de inundación, cuando no, a instalar las edificaciones directamente en el fondo de esos cauces fluviales y desdibujar por completo el trazado original de los mismos. Esta forma de expansión urbana delata una grave situación de negligencia por parte de las administraciones locales al haber permitido este tipo usos en zonas de inundación frecuente. 
Figura 6. Porcentaje del total de superficie construido en la zona inundable de cada periodo de retorno.

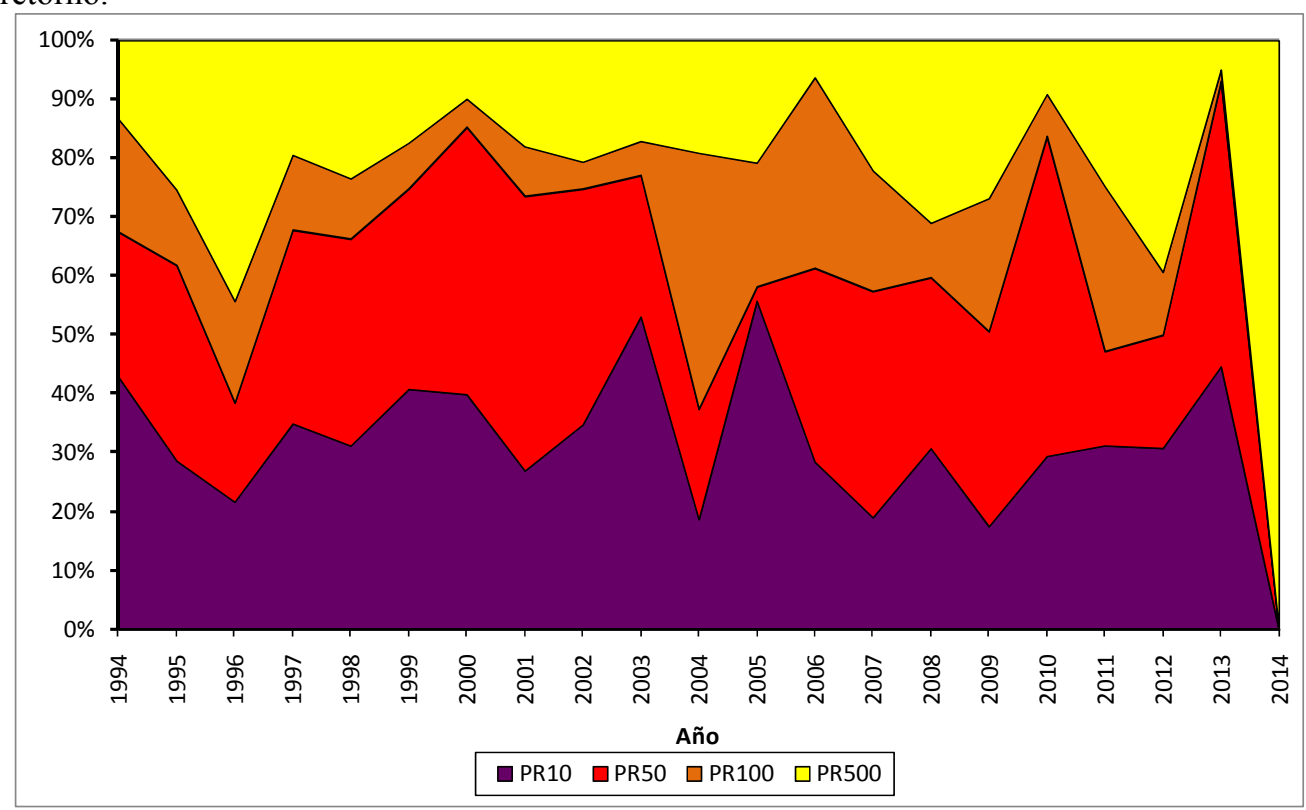

Fuente: Elaboración propia.

Figura 7 y 8 . Evolución de la superficie e inmuebles construidos en zona expuesta y no expuesta al peligro de inundación (1994-2014). Valores normalizados.
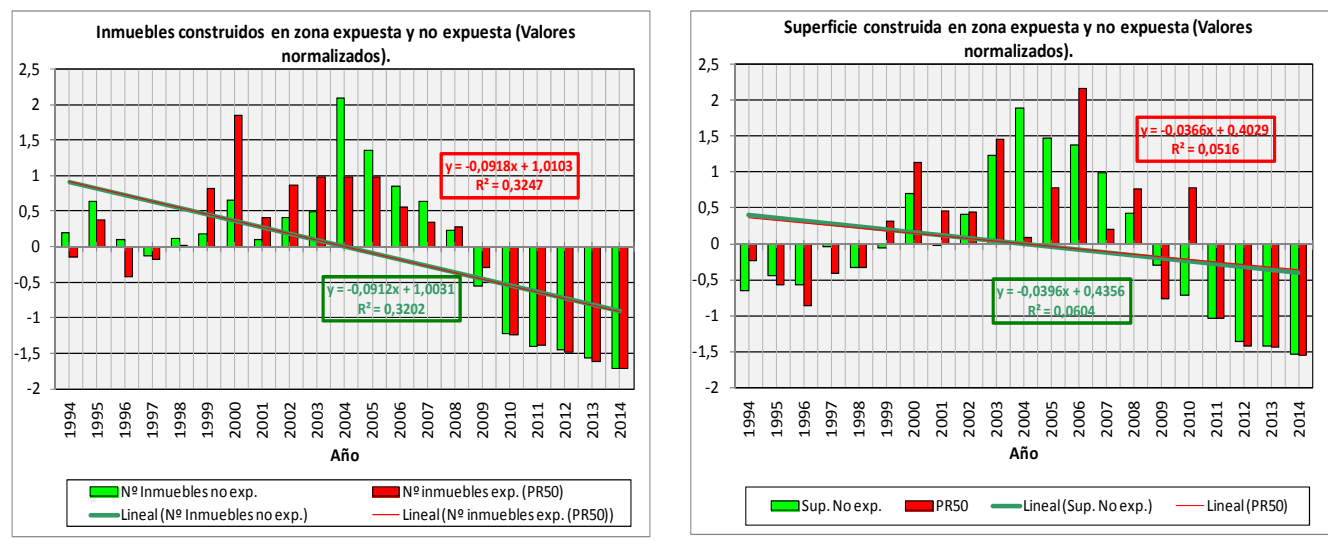

Fuente: Elaboración propia. 
Por último, las figuras 7 y 8 muestran las series normalizadas de superficies construidas y número de inmuebles en zona expuesta del PR50 y no expuesta. El análisis de dicha zona inundable sin incluir a los PR100 y 500 se justifica porque el PR50 es el periodo que comúnmente suele presentar una mayor restricción de usos tanto en las políticas de ordenación como en las urbanísticas. De la interpretación de ambos gráficos destaca, como los valores de ambas series (tanto lo expuesto como lo no expuesto en inmuebles como en superficie) han descendido a tenor del contexto económico vivido en el periodo analizado, sin embargo, resulta preocupante que el comportamiento de las dos no sea desigual, lo que pone de manifiesto el reducido papel o aplicabilidad de las medidas de protección de cauces contenidas en las DPOTL. En otras circunstancias estaríamos hablando de dos tendencias dispares en las que se advertirían evoluciones decrecientes de lo expuesto y no como sucede actualmente que, incluso tras la aprobación de las DPOTL existen años que, ya sea en superficie o en número de inmuebles, el valor proporcional de lo expuesto prevalece sobre el no expuesto.

\section{DISCUSIÓN Y CONCLUSIONES ${ }^{3}$}

La conclusión más evidente del presente estudio es la importante conversión a zonas expuestas de una superficie creciente y que viene acumulándose desde el inicio del periodo analizado. Esta ocupación de zonas inundables ha seguido los ritmos de la coyuntura económica de esas últimas décadas pese a la aprobación de los instrumentos de ordenación que se suponían preparados o al menos diseñados para organizar un proceso de ocupación adaptativo. La tendencia constructiva tanto en zonas expuestas como no expuestas no ha conseguido desligarse en sus patrones de comportamiento por lo que se podría afirmar que las medidas de limitación incluidas en las DPOTL y en otras legislaciones con competencia en la materia (ver Ley Suelo 1998, 2008, Directriz Básica de Planificación de Protección Civil ante el Riesgo de Inundaciones y las específicas de los PGMOU interesados) han ejercido un papel ineficiente que ni siquiera han ejercido labor alguna ni en los momentos de menor presión urbanística de zonas susceptibles de ser inundadas tras el estallido de la crisis financiera actual.

Habría que buscar las explicaciones en: la demora en la implementación definitiva de las medidas de limitación de usos en los planes generales de ordenación urbana municipal que las DPOTL regulan; por solapamiento de competencias entre administraciones o, simplemente, una combinación de las anteriores junto al deficiente papel de las administraciones competentes en ordenación territorial que, dejándose llevar por los intereses económicos derivados de esta ocupación caótica de

3 Agradecimientos; Al profesor Francisco Calvo García-Tornel, por su incasable y desinteresado apoyo en todo nuestro trabajo. 
territorios expuestos al peligro (Romero et al., 2012), han obviado el cometido principal que legitima su función, esto es, según Giddens (2002), mitigar el riesgo a las poblaciones.

Una vez constatada esa realidad, y a la vista que, hasta el momento presente, no resulta concluyente el aumento natural de los episodios de precipitación susceptibles de provocar inundaciones (Benito et al., 2005; Turco y Llasat, 2011; Gallego et al, 2011; Benito y Machado, 2012), en base a los resultados de exposición obtenidos podemos afirmar que el aumento del riesgo de inundación en el periodo y ámbito geográfico analizado se debe más en esta área a la acentuación de la vulnerabilidad y la exposición, que a un aumento de la peligrosidad. Los resultados del presente estudio coinciden con los que ya en su día apuntaban la Directiva Europea (DIRECTIVA 2007/60/CE, Parlamento Europeo, 2007) y el IPCC (2012), que señalan al incremento de los asentamientos humanos y los bienes económicos en las llanuras aluviales como uno de los factores principales que han contribuido a elevar las probabilidades de inundaciones en el mundo, así como su impacto negativo (Easterling et al, 2000; Changnon et al. 2000; Bouwer et al. 2007, Barredo, 2009; IPCC 2012). Por tanto, parece más lógico pensar que cada vez que se produce una inundación con efectos dañinos la responsabilidad haya que buscarla precisamente en la negligencia por parte de las administraciones regionales y locales al haber permitido este tipo usos en zonas de inundación frecuente.

\section{BIBLIOGRAFÍA}

BALASCH, J.C., MONSERRATE, A., SÁNCHEZ, A., ABELlÀ A, TUSET J, LLUÍS J y BARRIENDOS M (2014). Analysis of the major floods in the Ebro River basin (Iberian Peninsula) since 1600 AD. En EGU General Assembly Conference Abstracts (Vol. 16, p. 5733).

BARREDO, J.I. (2009). Normalized flood losses in Europe: 1970-2006. Natural Hazards and Earth System Science, 9(1), 97-104.

BARREDO, J.I., SAURI, D. y LLASAT, M.C. (2012). Assessing trends in insured losses from floods in Spain 1971-2008. Natural Hazards and Earth System Science, 12 (5), 1723-1729.

BARRIENDOS, M., BALASCH, J.C., TUSET, J. AND LLUÍS J. (2014). The Prediflood database. A new tool for an integrated approach to historical floods in Catalonia (NE Iberian Peninsula), AD 1033-2013. En EGU General Assembly Conference Abstracts, vol 16, 5907.

BENITO, R., y MACHADO, M.J. (2012). Floods in the Iberian Peninsula. En Kundzewicz, Z.W. (Ed): Changes in flood risk in Europe, Wallingford, IAHS Press and CRC Press/Balkema, 372-383.

BENITO, G., BARRIENDOS, M., LLASAT, C., MACHADO, M., у THORNDYCRAFT, V. (2005). Impacts on natural hazards of climatic origin. En Moreno, J.M. (Ed.): A Preliminary Assessment of the Impacts in Spain due to the Effects of Climate Change, Madrid, Ministerio de Medio Ambiente, 507-528. 
BOCHET, E. y GARCÍA-FAYOS, P. (2004). Factors controlling vegetation establishment and water erosion on motorway slopes in Valencia, Spain. Restoration Ecology, 12 (2), 166-174.

BOUWER, L.M., CROMPTON, R.P., FAUST, E., HOPPE, P., y PIELKE JR., R.A. (2007). Disaster Management: Confronting Disaster Losses, Science, 318, 753.

BURRIEL, E. (2008). La década prodigiosa del urbanismo español (1997-2006). Scripta Nova, 12(270), 64.

CUTTER, S. L., BORUFF, B. J., y SHIRLEY, W. L. (2003). Social vulnerability to environmental hazards. Social science quarterly, 84(2), 242-261.

CARDONA, O.D, VAN AALST, M.K., BIRKMANN, J. FORDHAM, M. MCGREGOR, G., PÉRES, R. PULWARTY, R.S., SCHIPPER, E.L.F. y SINH, B.T. (2012). Determinants of risk: exposure and vulnerability. En: Field CB et al. (eds) Managing the Risks of Extreme Events and Disasters to Advance Climate Change Adaptation. A Special Report of Working Groups I and II of the Intergovernmental Panel on Climate Change (IPCC). Cambridge, UK, and New York, NY, USA, Cambridge University Press, 65-108

CHANGNON, S.A., PIELKE JR, R.A., CHANGNON, D., SYLVES, R.T., y PULWARTY, R. (2000). Human Factors Explain the Increased Losses from Weather and Climate Extremes. Bulletin of the American Meteorological Society, 81(3), 437-442.

EASTERLING, D.R., MEEHL, G.A., PARMESAN, C., CHANGNON, S.A., KARL, T.R. y MEARNS, L. O. (2000): Climate extremes: observations, modeling, and impacts. Science, 289(5487), 2068-2074.

EUROPEAN COMMISSION (EC) (2007). Directive 2007/60/CE of the European Parliament and of the Council on the assessment and management of flood risks. 23 October 2007.

GAJA, F. (2008). El "tsunami urbanizador" en el litoral mediterráneo. El ciclo de hiperproducción inmobiliaria 1996-2006. Scripta Nova, 12(270), 66.

GALLEGO, M.C., TRIGO, R.M., VAQUERO, J.M., BRUNET, M., GARCÍA, J.A., SIGRÓ, J., y VALENTE, M.A. (2011). Trends in frequency indices of daily precipitation over the Iberian Peninsula during the last century. Journal of Geophysical Research: Atmospheres (1984-2012), 116 (D2).

GARCIA, F.M. (2013). Una metodología para la delimitación y clasificación de las formas urbanas en las ciudades intermedias españolas durante el siglo XX basada en SIG. V Seminario Internacional de Investigación en Urbanismo, BarcelonaBuenos Aires, junio 2013. Barcelona, 1199-1215.

GIDDENS A (2002). Runaway world: How globalisation is reshaping our lives. London, Profile books.

GIL-GUIRADO, S., PÉREZ, A., LÓPEZ, F. y BARRIENDOS, M. (2014). Review of flood alert thresholds based on the socioeconomic impact of catastrophic events in the southeastern Spain. EMS Annual Meeting Abstracts Vol. 11, 14th EMS / 10th ECAC.

HOOKE, J.M. (2006). Human impacts on fluvial systems in the Mediterranean region. Geomorphology, 79 (3), 311-335. 
IPCC (2012). Managing the Risks of Extreme Events and Disasters to Advance Climate Change Adaptation. A Special Report of Working Groups I and II of the Intergovernmental Panel on Climate Change [Field, C.B., V. Barros, T.F. Stocker, D. Qin, D.J. Dokken, K.L. Ebi, M.D. Mastrandrea, K.J. Mach, G.-K. Plattner, S.K. Allen, M. Tignor, y P.M. Midgley (eds.)]. Cambridge y New York, Cambridge University Press, 582.

JIMÉNEZ, F. (2009). Building boom and political corruption in Spain. South European Society and Politics, 14(3), 255-272.

JONGMAN, B., KREIBICH, H., APEL, H., BARREDO, J.I., BATES, P.D., FEYEN, L., y WARD, P.J. (2012). Comparative flood damage model assessment: towards a European approach. Natural Hazards and Earth System Science, 12(12), 37333752.

JONGMAN, B., KOKS, E. E., HUSBY, T. G. y WARD, P. J. (2014). Financing increasing flood risk: evidence from millions of buildings. Natural Hazards and Earth System Sciences Discussions, 2 (1), 137-165.

LLASAT, M.C., LÓPEZ, L., BARNOLAS, M. y LLASAT-BOTIJA, M. (2008). Flash-floods in Catalonia: the social perception in a context of changing vulnerability. Advances in Geosciences, 17 (17), 63-70.

MAGRAMA. (05 de Abril de 2014). Sistema Nacional de Cartografía de Zonas Inundables. http://www.magrama.gob.es/es/agua/temas/gestion-de-los-riesgos-deinundacion/snczi/

MHAP. (08 de Octubre de 2014). Dirección General del Catastro. http://www.sedecatastro.gob.es/

MOEL, H.D., ALPHEN, J.V., y AERTS, J.C. (2009). Flood maps in Europe-methods, availability and use. Natural Hazards and Earth System Science, 9(2), 289-301.

OLCINA, J. (2007). Riesgo de inundaciones y ordenación del territorio en España. La consideración del peligro de inundaciones en los procesos de planificación territorial. Alicante, Fundación Instituto Euromediterráneo del Agua.

PÉREZ, A. (2007). Cuestiones Medioambientales y Ordenación del Territorio en el Litoral de la Región de Murcia. Murcia, Asociación Murciana de Ciencia Regional y Caja de Ahorros del Mediterráneo.

ROMERO, J., JIMÉNEZ, F. y VILLORIA, M. (2012). Bubble in Spain (1996-2010) and its territorial, environmental, and sociopolitical consequences. Environment and Planning C: Government and Policy, 30, 467-486.

SYMEONAKIS, E., CALVO-CASES, A. y ARNAU-ROSALEN, E. (2007). Land use change and land degradation in southeastern Mediterranean Spain. Environmental management, 40 (1), 80-94.

TURCO, M. y LLASAT, M.C. (2011). Trends in indices of daily precipitation extremes in Catalonia (NE Spain), 1951-2003. Natural Hazards Earth System Sciences, 11, 3213-3226.

VERA REBOLLO, J. F. y ESPEJO MARÍN, C. (2006). El papel de los instrumentos de planificación en las dinámicas productivas y territoriales: las directrices y el plan de ordenación del litoral de la Región de Murcia. 